\title{
Editorial
}

\section{Developmental Origins of Obesity: A Critical Periods Perspective}

The developmental origins of obesity perspective examines the potential associations among a compromised fetal environment, accelerated postnatal growth, and/or early "environmental" stimuli on fuel utilization/tissue partitioning and excessive fat mass accrual in childhood. Indeed, the increasing rates of obesity, particularly in the pediatric population, disproportionately burdening some populations at a higher rate than others, cannot be explained without some genetic component. Accordingly, the genetic and epigenetic influences on "programming" of adipose tissue may provide insight into potential mechanisms driving fat accumulation early in the life course, with particular salience during critical periods of development (i.e. intrauterine, early postnatal, prepubertal, and adolescent). The extent to which excessive fat mass accumulation beginning in childhood is attributable to environmental and/or genetic factors remains uncertain due to the lack of understanding of biological and non-biological factors, their interaction, and the extent to which they confound each other. The success in identifying the etiology of pediatric obesity particularly among diverse populations will rely on the development of precise phenotypic measurements and sophisticated experimental designs that take into account the developmental origins and trajectory of body composition as well as the social, cultural and economic context that influence them. This supplement provides reviews and original research highlighting important scientific contributions upon examination of the developmental origins of obesity.

Beginning in utero, the programming that occurs is a feature of the plasticity of tissues. It is very possible that each of the critical periods during growth and development has an additive effect on obesity status through genetic, behavioral, or physiologic mechanisms. Chandler and Bush review studies indicating adiposity at birth is intricately linked to maternal metabolic health, particularly glucose tolerance, and in turn, is likely to be involved in prenatal programming of obesity among neonates. In the postnatal period, however, studies on future obesity risks are generally centered on birth weight, as research has consistently linked birth outcomes to risk for chronic diseases, with both low and high birth weight indicative of risk. Willig and colleagues report that birth weight was positively associated with adiposity and fat-free mass among children aged 7 to 12 years. The analysis included a combination of biological and environmental covariates in the model allowing a more accurate assessment of the association of birth weight with body composition parameters. Interestingly, the effect was attenuated by physical activity levels, suggesting an environmental contribution that may override "programming" effect. Kagawa and Hills evaluate the increments in height and weight over the 50-year period among Japanese children. The analysis is concordant with that which has been previously observed; the increments in height and weight from 1950 to 1960 were rapid compared to the rest of the survey period, which can be explained by acceleration in the tempo of growth. The study revealed that the age of peak height velocity for all assessed variables decreased rapidly for about a year during the period of 1950 to 1960 , reflective of the recovery of many Japanese children from a malnourished state during World War II. After accounting for these factors, 
increments in body weight were small and the subsequent risk of childhood obesity may not have increased as rapidly as has been suggested among Japanese children, though results suggest that increases in weight and BMI may be due to lifestyle factors. Nevertheless, the authors suggest that due to potential for higher body weight in younger age groups and the increasingly sedentary lifestyle of many Japanese, preventative strategies are recommended to minimize childhood obesity and related metabolic complications.

Furthermore, childhood obesity prevention strategies may be of considerable importance as children enter the pubertal transition. During adolescence, interactions of reproductive and metabolic hormones contribute to body weight control and when aberrant, play a role in the pathogenesis of obesity. Given the roles of the hypothalamic-pituitary-adrenal (HPA) and hypothalamic-pituitary-gonadal (HPG) axes (which are not fully mature until mid-puberty) in energy homeostasis, small changes in the development of neuroendocrine pathways occurring during the pubertal transition have repercussions for a wide range of basic physiological and metabolic functions that influence energy balance. Cardel and colleagues investigate five putative contributors to pediatric obesity beyond the "Big Two" (diet and physical activity) that have recently been documented to be associated with fat accumulation and energy imbalance in adolescence. The authors suggest that fluctuations in the hormonal milieu during puberty may "trigger" malprogramming of metabolism as a result of influences occurring in utero or in early childhood yet not realized until puberty. Further, they propose potential contributions of endocrine disruptors, child feeding practices, stress and/or the built environment worthy of consideration in disentangling the etiology of pediatric obesity.

Paramount to designing effective intervention and prevention strategies in the pediatric population is reliable and accurate methodology to quantify regional fat distribution and an understanding of the association between adipose tissue distribution and health risks. Children's fat partitioning is different from that of adults. Advances in MRI have made it possible for future studies to investigate sub-depots. Although adipose tissue sampling in the omental and mesenteric adipose tissue compartments has greatly improved our understanding of the mechanisms at the cellular and molecular levels, biopsy of these adipose tissue depots is likely to be limited to morbidly obese subjects who undergo bariatric surgery. Imaging methods may be utilized to fill in gaps when studying these adipose tissue depots in non-obese subjects or subjects in the very early stages of obesity.

Evidence of a considerable genetic component to individual differences in weight at birth and BMI at all periods in childhood are reviewed by Rokholm, Anderson and Sorensen. Some studies suggest an age trend in heritability and the importance of shared environmental factors. Although the focus in genetic epidemiological research in the recent years has moved to molecular genetics studies, quantitative genetic methods undoubtedly still have more to offer. Twin and family studies show that the majority of variation in BMI is caused by genetic factors. Nevertheless, studies on candidate genes have only accounted for a few percentages of the total genetic variation, calling for new ways of understanding the role of genes in obesity development. Throughout this exploratory phase, twin and family studies are ideal, since hypotheses can be tested without restricting the analyses to particular genetic loci. More research using the twin and family approach should therefore be encouraged. 
Several years ago, using animal models, it was proposed that stimuli during intrauterine and early life could influence metabolic imprinting of networks in the offspring, perpetuating or even amplifying obesity susceptibility. More recent studies in animals and humans demonstrate that hypothalamic maturation postnatally is dependent on cues from peripheral signals (i.e., leptin, insulin, GH, IGF-1, etc). Alterations in these signaling pathways result in failure to form hypothalamic connections necessary for normal energy homeostasis. Although plasticity is a finite characteristic, stimuli during critical periods could interfere with the neuroendocrine regulation of proliferation or the differentiation of tissues and/or exert effects on genes. Exposures even during a brief period can alter long-term gene expression and compromise normal physiology and metabolism. It is evident, however, that a number of factors in hypothalamic maturation and resultant influence on energy homeostasis at critical periods of development has a profound influence on pathways involved in tissue partitioning and responsiveness to homeostatic signals and tissue function in childhood.

Krista R. Casazza (Guest Editor)

Department of Nutrition Sciences and Clinical Nutrition Research Center University of Alabama at Birmingham Birmingham, AL, USA Tel: (205) 975-6226

Fax: (205) 934-7050

E-mail: kristac@uab.edu

(C) Krista R. Casazza; Licensee Bentham Open.

This is an open access article licensed under the terms of the Creative Commons Attribution Non-Commercial License (http: //creativecommons.org/licenses/by$\mathrm{nc} / 3.0 /$ ), which permits unrestricted, non-commercial use, distribution and reproduction in any medium, provided the work is properly cited. 